\title{
The structure of smart fluids
}

\section{Whittle and W. A. Bullough}

ELECTRO-RHEOLOGICAL fluids, which can be transformed into a gel-like state by applying an electric field, are the subject of increasing interest in engineering sciences, yet little is known of the microscopic basis of their remarkable properties. Tao and colleagues, at the Southern Illinois University, have devised a laser-diffraction technique for revealing the microscopic structures of such fluids ${ }^{1}$, and confirm the arrangement of the electrified fluids predicted by their models.

Among the most spectacular of 'smart' materials, electro-rheological fluids are slurries of particles suspended in an insulating fluid. On application of a strong electric field, perhaps 1 megavolt per metre, they become plastic in the sense of exhibiting a yield stress. The rheological changes are associated with the formation of chains and fibrils of the suspended particles under the influence of the applied field ${ }^{2}$. But it is hard to see what is going on at the volume fractions (20-40 per cent) with which useful rheological changes occur, because of the opacity of the fluids. Instead, observations are typically made with dilute solutions (around 2 per cent volume fraction), in which the chains can be seen to coalesce into columns (see figure) and even (at higher concentrations) into extended three-dimensional structures.

The mechanism of this behaviour is normally discussed in terms of particle polarization $^{2,3}$ although this is not yet quantitatively established as the only mechanism and other effects may be present. In earlier work, Tao and $\mathrm{Sun}^{4}$ predicted a body-centred tetragonal (b.c.t.) lattice structure for the columns on the basis of energy minimization for a system of induced dipoles. This is related to the better known body-centred cubic but only two sides of the unit cell are equivalent in the primitive lattice.

Tao and colleagues have now devised a novel laser-diffraction method which confirms that the column structure is body-centred cubic at a volume fraction of 20 per cent. At these concentrations light scattering is useless because of the high degree of multiple scattering. The new method relies on the use of a suspension of glass microspheres of highly uniform diameter. Although glass spheres do not give very good electrorheological fluids (in terms of the yield stress achievable), we may expect them to produce representative structures.

The resulting diffraction pattern for the fluid subjected to a field is not related to any conventional diffraction pattern and the authors propose that it is the result of multiple lensing by particles arranged in a regular lattice. A textbook analysis of a linear periodic array of focusing elements reveals that light can propagate along the lattice under certain circumstances and allows the prediction of the pattern imposed on the exit beam. The authors find that only the b.c.t. structure can account for the observed pattern.

There has always been considerable

\section{IMAGE UNAVAILABLE FOR COPYRIGHT REASONS}

Columns of dilute cornstarch in motor oil ( 5 per cent by mass) in a field of 2.5 megavolts per metre. Depth of figure, 365 $\mu \mathrm{m}$. (Courtesy of D. G. Froode.)

argument about the nature of the interaction in electro-rheological fluids and the fact that the predicted structure is revealed experimentally supports the case for dipole-dipole interactions. Nevertheless, it leaves ample scope for discussion as to how the dipoles arise in the first place - indeed this may differ from fluid to fluid. The nature of the structure also has important predictive potential, as the relation between physical properties and extended structure is well documented. Transport properties such as thermal and electrical conductivity follow the lattice symmetry and will therefore exhibit anisotropy. Mechanical properties like the yield stress will also be related and should exhibit preferential slip planes. Recent work into the use of relatively dilute fluids for display systems and anti-radiation films could directly benefit from an understanding of the anisotropy, as these devices operate under static conditions and use relatively dilute suspensions. In this case, as in many applications, a rapid response of the fluid is crucial and the dynamic aspect of structure formation is an area that this work could now extend to.

Any comparison between theory and experiment is welcome in this respect and it resolves an old question. But much remains to be done. There is a gulf between these kinds of experiments and the real world of engineering requirements. These are complicated systems and many experiments and theoretical approaches are understandably made far from the regime where electrorheological fluids are actually useful. The real fluids are composed of polydisperse irregularly shaped particles unlikely to settle into a regular lattice structure and there is no evidence as yet to suggest that the extra effort and cost involved in producing monodisperse systems will improve their useful properties.

In this context the stability of particles under hostile engineering conditions must also be considered. Electrorheological fluids are currently under investigation with a view to providing rapid coupling between components in high-speed machines (the gel-like state can be induced within hundreds of microseconds), and in these applications they are subjected to a shear field in addition to the electric field. The competitive edge offered by these devices is in the speed of response and this is strongly dependent upon particle concentration. Generally, volume fractions of 30 per cent upwards are necessary to produce viable devices. The response time is further influenced by the shear rate and temperature.

The shear rate in an operating electrorheological valve may be as high as $20,000 \mathrm{~s}^{-1}$, and for clutches centrifugal forces of around $300 \mathrm{~g}$ may arise $\mathrm{e}^{5}$. These conditions can cause considerable practical problems in the guise of sedimentation or plating out of the particle phase. Computer simulations suggest that even at relatively low shear rates the particles arrange themselves into sheets parallel to the flow ${ }^{6,7}$. It seems unlikely that such dynamic structures can be described with the simplicity that arises at zero shear rate, but the easier theoretical situation is related to how quickly the dynamic equilibrium is attained and decays in response to a changing applied field.

It is important to ask answerable questions and to give answers as excellent as Tao and colleagues have done. But to tackle the wider commercially important problems needs collaboration between workers who have access to materials properties, engineering-scale experimental data on time response and computer simulations.

M. Whittle and W. A. Bullough are in the Department of Mechanical Engineering and Process Control, University of Sheffield, Sheffield S1 3JD, UK.

\footnotetext{
1. Chen, T., Zitter, R. N. \& Tao, R. Phys. Rev. Lett. 68 2555-2558 (1992)

2. Tao, R. (ed.) Electrorheological Fluids (World Scientific, Singapore, 1992).

3. Block, H. \& Kelly, J. P. J. Phys. D 21, 1661-1677 (1988).

4. Tao, R. \& Sun, J. M. Phys. Rev, Lett. 67, 398-401 (1991)

5. Bullough, W. A. et al. Mechatronic Syst. Eng. (in the press).

6. Whittle, M. J. Non-Newtonian Fluid Mech. 37, 233263 (1990)

. Melrose, J. Molec. Phys. (in the press)
} 